Research Article

\title{
Computationally Efficient Compressed Sensing-Based Method via FG Nyström in Bistatic MIMO Radar with Array Gain-Phase Error Effect
}

\author{
Jurong Hu, Evans Baidoo (iD, Lei Zhan, and Ying Tian \\ College of Computer and Information Engineering, Hohai University, Nanjing, China \\ Correspondence should be addressed to Evans Baidoo; ebaidoo2@hhu.edu.cn
}

Received 5 August 2019; Revised 22 January 2020; Accepted 5 February 2020; Published 10 March 2020

Academic Editor: Muhammad Ramlee Kamarudin

Copyright @ 2020 Jurong Hu et al. This is an open access article distributed under the Creative Commons Attribution License, which permits unrestricted use, distribution, and reproduction in any medium, provided the original work is properly cited.

\begin{abstract}
In this paper, a robust angle estimator for uncorrelated targets that employs a compressed sense (CS) scheme following a fast greedy (FG) computation is proposed to achieve improved computational efficiency and performance for the bistatic MIMO radar with unknown gain-phase errors. The algorithm initially avoids the wholly computation of the received signal by compiling a lower approximation through a greedy Nyström approach. Then, the approximated signal is transformed into a sparse signal representation where the sparsity of the target is exploited in the spatial domain. Finally, a CS method, Simultaneous Orthogonal Matching Pursuit with an inherent gradient descent method, is utilized to reconstruct the signal and estimate the angles and the unknown gain-phase errors. The proposed algorithm, aside achieving closed-form resolution for automatically paired angle estimation, offers attractive computational competitiveness, specifically in large array scenarios. Additionally, the analyses of the computational complexity and the Cramér-Rao bounds for angle estimation are derived theoretically. Numerical experiments demonstrate the improvement and effectiveness of the proposed method against existing methods.
\end{abstract}

\section{Introduction}

Lately, the demanding requirements, the accuracies in location and the need for high resolutions for radar systems, have led to extensive attention to multiple-input multipleoutput (MIMO) radar [1]. The concept of the MIMO radar system is to instantaneously emit mutually orthogonal waveforms with multiple antennas while receiving the reflected echoes with multiple receive antennas. Many research works have indicated the effectiveness of MIMO radar in the suppression of noise, dominance over the effect of fading, enhanced spatial resolution, and improved parameter identifiability, among others [2-6]. Based on the transmit and receive location of the antenna, colocated MIMO radar can be categorized into monostatic and bistatic radar. Different from the monostatic MIMO, bistatic MIMO radar structures a widely separated antenna distance between the transmitter and receiver positions, hence its ability to achieve an improved performance of target localization and detection [7]. This paper studies the angle estimation problem, considering a bistatic MIMO where the angles to the transmit and receive arrays are regular and dissimilar; also referred to as direction of departure (DOD) and direction of arrival (DOA).

The angle estimation problem in array signal processing has received intense attention in recent times. For example, the classical peak searching methods, Multiple signal classification (MUSIC) and capon algorithm, have been studied by Zahernia et al. and Yan et al. [8, 9], respectively, although they result in high computational cost while Duo fang et al. [10] exploit the nonpeak searching capabilities of signal parameters via rotational invariance techniques (ESPRIT) approach to achieve angle estimation. It must be noted that these estimators present suitable results for target localization problems. Nonetheless, in practical radar fields, the presence of gain-phase errors existing between the antenna 
elements may occur due to incongruity between antenna arrays [11-13]. Thus, such imperfection of the array antennas will seriously degrade the estimation performance of the above-listed estimators. As such, many algorithms have been proposed to resolve the gain-phase inaccuracies between the antennas. In [14], an ESPRIT-like method is presented but requires an extra paring scheme. Then, Li et al. [15] proposed a propitious reduced-dimension MUSIC procedure to achieve angle and gain-phase error estimations. Unfortunately, these subspace methods do not consider the additional characteristics of the received signals and only resort to distinguishing the noise subspaces.

Compressed sensing- (CS-) based techniques have been proposed to improve angle estimation accuracies by the exploitation of the signal sparsity in the spatial domain [16-18]. The CS theory asserts that, with limited samples, a uniformly sampled signal at or above the Nyquist rate can be recovered [19]. CS-based methods have proven to present better performance even with a low number of snapshots and signal-to-noise ratio in addition to estimation accuracies for correlated targets than the traditional methods [20-22]. In [23], the CS sparse representation methods, orthogonal matching pursuit method (OMP), multiple OMP, optimised OMP [24], and simultaneous OMP (SOMP) [25] were proposed to resolve the angle estimation problem at the expense of low computation. The fast iterative shrinkagethresholding algorithm (FISTA) [26] and the CS sparse Bayesian learning [27] have also been applied to realize high target resolution. Then, Liu et al. [28] put forward a sparsebased model to tackle the arbitrary array errors introduced by unknown phase errors. In [29], an iterative CS method is proposed to resolve the mutual coupling between antenna arrays. However, the methods in [24-29] suffer from high computational complexity and hence difficult to apply in practical scenarios.

Different from others, we propose a robust algorithm that obtains the angle and antenna gain-phase error estimation for the bistatic MIMO radar. The proposed algorithm which exploits the target sparsity to improve the estimation performance maintains a high accuracy with a very low computational load. Our method is based on the compressed sense theory via a fast greedy (FG) selection Nyström approach with an incorporated gradient descent procedure for gain-phase error estimation.

The Nyström method, earlier adopted by [30] for kernel matrix sparsification, is an extensively exploited approach known to speed up algorithms [31, 32]. For instance, Qian et al. [33] applied the kernel matrix approximation to achieve low computation and better DOA estimation.

To continue, by formulating a diagonal matrix to describe the effect of the antenna array errors, the theoretical expression of the estimation of the gain-phase error is computed using the gradient descent method following signal reconstruction through a CS-based SOMP method. The performance of the proposed algorithm is compared with current methods within the system model implemented. In addition, the theoretical derivation of the Cramér-Rao bound (CRB) of the proposed method is analysed. The proposed algorithm is robust against array errors and presents an improved computationally efficient closed-form solution to automatically paired angle estimation. Results carried out from numerical simulation proved the efficiency of the proposed method.

The remainder of the paper is ordered as follows: Section 2 presents the data model of the bistatic MIMO radar with gain-phase errors, followed by the proposed sparse algorithm via the Nyström approximation and the gain-phase derivation in Section 3. Section 4 deals with the derivation of the Cramér-Rao bound (CRB) for the proposed model alongside the analyses of the computational complexity. Section 5 details the experimental results to verify the effectiveness of the proposed method then followed by the final Section 6, which summarises the paper.

Notations. vectors take on small letters in bold face (e.g., a) while matrices take on bold face in capital letters (e.g., A). An identity matrix $N \times N$ is represented as $I_{N} \cdot \mathscr{C} \mathcal{N}(g, \mathbf{H})$ is the complex Gaussian distribution, where $g$ denotes the mean and $H$ denotes the variance. $\|\cdot\|_{1},\|\cdot\|_{2},\|\cdot\|_{F}, \operatorname{vec}\{\cdot\}$, $\operatorname{Tr}\{\cdot\}, \otimes, \odot,(\cdot)^{H},(\cdot)^{*}, \dagger$, and $(\cdot)^{T} z$ indicate $\ell_{1}$ norm, $\ell_{2}$ norm, Frobenius norm, matrix vectorization, the trace of a matrix, Kronecker product, Khatri-Rao product, Hermitian transpose, matrix conjugate, pseudoinverse, and the transpose of the matrix, respectively. $\mathbf{B},[\mathbf{B}]_{q}$ represents the $q$-th column of the matrix $\mathbf{B}$ whereas $\mathbf{b},[\mathbf{b}]_{q}$ represents the $q-$ th entry of a vector b. $\mathscr{R}\{\cdot\}$ and $I\{\cdot\}$ are designated for the real and imaginary parts of the complex, respectively, and $\mathbb{E}[\cdot]$ denotes mathematical expectation.

\section{Data Model}

A bistatic MIMO radar system, narrow-banded forming an $M$ transmit and $N$ receive omnidirectional array elements, with a fundamental spacing distance, $d$ of half-wavelength, and uniform linear arrays as shown in Figure 1 is considered. All transmitting antenna, $(m=1,2, \ldots, M)$ transmit varying coded constant periodic signals over $(p=1,2, \ldots, P)$ pulses with independent and identical bandwidth and center frequency to target and collected by the $(n=1,2, \ldots, N)$ receive antenna elements simultaneously. Consider that, there exist $K$ independent targets that appear from varying propagation paths but are in the same surveillance region far further than the transmit and receive antennas. The direction of the $k$ th target is denoted as $\left\{\left(\varnothing_{k}, \theta_{k}\right)\right\}_{k=1}^{K}$ where $\varnothing_{k}$ and $\theta_{k}$ signify the DOD and DOA of target, respectively. Then, the output of the signal received after match filtering is expressed as follows:

$$
\mathbf{h}(t)=\left[\mathbf{a}_{m}\left(\varnothing_{k}\right) \otimes \mathbf{b}_{n}\left(\theta_{k}\right)\right] \bar{s}(t)+\mathbf{e}(t),
$$

where $\bar{s}(t)=\left[\bar{s}_{1}(t), \bar{s}_{2}(\mathrm{t}), \ldots, \bar{s}_{K}(\mathrm{t})\right]^{T} \in C^{K \times 1}$ is the row vector of the target amplitude, with $\bar{s}_{k}=b_{k} e^{-j 2 \pi f_{k} t}$ where $b_{k}$ denotes the reflection coefficient and $f_{k}$ as the Doppler frequency. $\mathbf{e}(t) \in C^{M N \times 1}$ represents the additive noise component vector that follows $\mathscr{C} \mathscr{N}\left(0, \sigma^{2}\right) . \mathbf{a}_{m}\left(\varnothing_{k}\right) \triangleq$ $\left[1, e^{-j 2 \pi(d / \lambda) \sin \varnothing_{k}}, \ldots, e^{-j 2 \pi(d / \lambda) \sin \varnothing_{k}(M-1)}\right]^{T}$ is the transmit

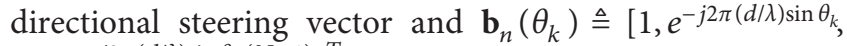
$\left.\ldots, e^{-j 2 \pi(d / \lambda) \sin \theta_{k}(N-1)}\right]^{T}$ is the receive directional steering vector with $\lambda$ as the carrier wavelength. The signal model 


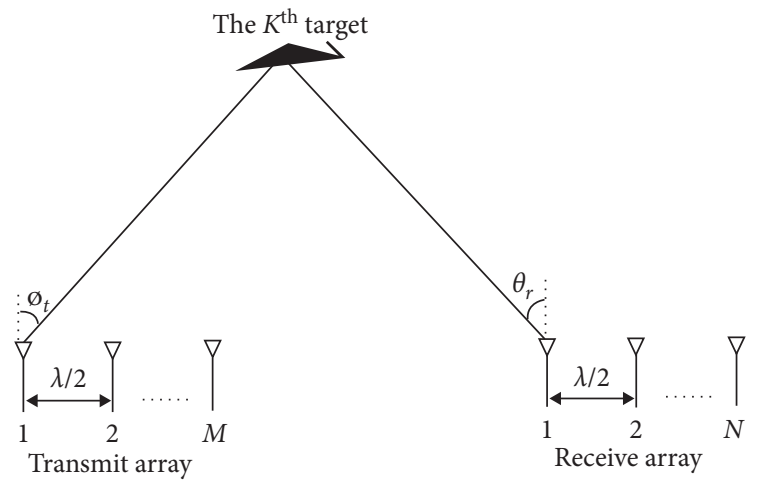

Figure 1: The bistatic MIMO radar system scenario.

representation in equation (1) is considered under ideal situation, but in practical radar array fields, antenna arrays can be affected by gain-phase errors. Thus, considering the gain-phase errors, the received signal can be rewritten as follows [13]:

$$
\widehat{h}(t)=\left[\widehat{a}_{m}\left(\varnothing_{k}\right) \otimes \widehat{b}_{n}\left(\theta_{k}\right)\right] \bar{s}(t)+\mathbf{e}(t),
$$

where $\widehat{a}_{m}\left(\varnothing_{k}\right)=\Gamma_{t} \mathbf{a}_{m}\left(\varnothing_{k}\right)$ and $\widehat{b}_{n}\left(\theta_{k}\right)=\Gamma_{r} \mathbf{b}_{n}\left(\theta_{k}\right)$ with $\Gamma_{t}$ and $\Gamma_{r}$ as the unknown gain-phase error matrices among the transmit array elements and receive array elements, respectively. We define the gain-phase errors as follows [15]:

$$
\begin{array}{r}
\Gamma_{t} \triangleq \operatorname{diag}\left\{\gamma_{t}\right\}, \gamma_{t}=\left[1, \ldots, 1, \varsigma_{1}^{t}, \ldots, \varsigma_{M-m}^{t}\right] \in C^{M \times M}, \\
\Gamma_{r} \triangleq \operatorname{diag}\left\{\gamma_{r}\right\}, \gamma_{r}=\left[1, \ldots, 1, \varsigma_{1}^{r}, \ldots, \varsigma_{N-n}^{r}\right] \in C^{N \times N}, \\
\Gamma=\left[\Gamma_{t} \otimes \Gamma_{r}\right],
\end{array}
$$

where $\Gamma$ is the gain-phase error matrix. When all receiver signals are accumulated into $P$ pulses, the outcome results in the signal model as

$$
\begin{aligned}
& \mathbf{H} \triangleq \hat{X} \boldsymbol{\beta}^{\mathbf{T}}+\mathbf{E}=\left[\Gamma_{r} \mathbf{B}_{N} \odot \Gamma_{t} \mathbf{A}_{M}\right] \bar{S}^{T}+\mathbf{E}, \\
& \widehat{X} \triangleq\left[\Gamma_{r} \mathbf{B}_{N} \odot \Gamma_{t} \mathbf{A}_{M}\right] .
\end{aligned}
$$

Similarly, (4) can be reexpressed as follows:

$$
\begin{aligned}
& \mathbf{H} \triangleq \Gamma \mathbf{X} \boldsymbol{\beta}^{\mathrm{T}}+\mathbf{E}=\left[\Gamma_{t} \otimes \Gamma_{r}\right]\left[\mathbf{B}_{N} \odot \mathbf{A}_{M}\right] \bar{S}^{T}+\mathbf{E}, \\
& \mathbf{X} \triangleq\left[\mathbf{B}_{N} \odot \mathbf{A}_{M}\right],
\end{aligned}
$$

where $\mathbf{H} \triangleq[\mathbf{h}(1), \mathbf{h}(2), \ldots, \mathbf{h}(P)] \in C^{M N \times P}, \bar{S} \triangleq[\bar{s}(1), \bar{s}(2)$, $\ldots, \bar{s}(P)]^{T} \in C^{P \times K}, \mathbf{A}_{M} \triangleq\left[\mathbf{a}_{m}\left(\varnothing_{1}\right), \ldots, \mathbf{a}_{m}\left(\varnothing_{K}\right)\right] \in C^{M \times K}$, and $\mathbf{B}_{N} \triangleq\left[\mathbf{b}_{n}\left(\theta_{1}\right), \ldots, \mathbf{b}_{n}\left(\theta_{K}\right)\right] \in \mathcal{C}^{N \times K}$, and $\mathbf{E} \triangleq[\mathbf{e}(1)$, $\mathbf{e}(2), \ldots, \mathbf{e}(P)] \in \complement^{M N \times P}$ is the Gaussian white noise matrix which is uncorrelated with the received signal. From the expression in (5), the angle estimation system model with array gain-phase errors is formulated. Hence, considering the existence of array errors in both transmit and receive antennas, a robust algorithm is developed to achieve DOD/ DOA estimation in $\mathbf{X}$ from the obtained signal, $\mathbf{H}$, without prior knowledge of the gain-phase errors.

\section{CS-Based Algorithm via FG Nyström Method}

In this section, to ensure the robustness of the algorithm and to obtain a relaxed computation of the DOD/DOA estimation from the erroneous rigid measurement matrix, a lower rank approximation of the received signal is initially derived before transforming the measurement matrix to the sparse domain where the gain-phase errors are estimated using a gradient descent approach. Finally, the sparse signal is reconstructed by exploiting the target sparsity to achieve paired angle estimation.

3.1. Signal Matrix Approximation by FG Nyström Method. Let the data matrix $\mathbf{H}=\left[\mathbf{h}_{1}, \mathbf{h}_{2}, \ldots, \mathbf{h}_{P}\right]$ be disintegrated into the form expressed as follows:

$$
\mathbf{H}=\left[\mathbf{H}_{1}, \mathbf{H}_{2}\right]^{T}
$$

By applying the Nyström covariance estimator, we seek to derive a close low-rank approximation of $\mathbf{H}$, which will circumvent the computation of the bulky sample covariance matrix (SCM) as well as its singular value decomposition. Since the data matrix of $\mathbf{H}$ is large, let $Q \in\{1,2, \ldots, M N\}$ be a parameter selected to divide the dimension of the received data matrix into submatrices. Then, the covariance matrix of $\mathbf{Y}=\mathbb{E}\left[\mathbf{H H}^{H}\right]$ can be disintegrated as follows:

$$
\mathbf{Y}=\left[\begin{array}{ll}
\mathbf{Y}_{11} & \mathbf{Y}_{12} \\
\mathbf{Y}_{21} & \mathbf{Y}_{22}
\end{array}\right]
$$

where $\mathbf{Y}_{11}, \mathbf{Y}_{12}, \mathbf{Y}_{21}$, and $\mathbf{Y}_{22}$ denote the $\mathbf{Y}$ signal submatrices. But it should be noted that the efficiency of the Nyström approximation is highly dependent on the subset of rows used, and thus, an optimum selection to achieve accurate estimation is required. Hence, in order to achieve optimal results, we proposed that the selection of $Q$ must be based on the insurance that $\mathbf{Y}_{11}$ must be of full rank and that the $Q$ value selection must be efficient enough to ensure minimal computational efficiency and optimal enough to maintain estimation accuracy. However, we cannot arbitrarily settle on any $Q$ value for array division to achieve an accurate estimation. Hence, we propose an FS solution by picking the row that keeps the best rank-1 approximation of $\mathbf{Y}$ matrix while ensuring that it is equivalent to $(M+N)$ in the worst case. Fast forward the objective falls on selecting the best row $q \in\{1,2, \ldots, Q\}$ from the rows of $\mathbf{Y}$ that ultimately uphold the criteria. The expression for a row candidate, $q$, of matrix $\mathbf{Y}$ for the rank-1 approximation [34] of the row is written as follows:

$$
\widehat{Y}_{\{q\}}=\mathbf{Y}_{q:} \mathbf{Y}_{q q}^{-1} \mathbf{Y}_{q:}^{H},
$$

where $\mathbf{Y}_{q \text { : }}$ designates the $q$-th row of $\mathbf{Y}$ and $\mathbf{Y}_{q q}$ is designated for the $q$-th diagonal element of $\mathbf{Y}$. Accordingly, (8) can be reexpressed as an optimization problem as follows:

$$
Q=\underset{q}{\operatorname{argmax}}\left\|\mathbf{Y}_{q:} \frac{1}{\sqrt{\mathbf{Y}_{q q}}}\right\|^{2}
$$

$$
\text { s.t. } \quad M+N \leq Q \leq \min (M N, P) \text {. }
$$

In particular, the Q-th selection is the selection with the highest variance sufficient to guarantee optimum estimation. The choice of $Q$ has a computational complexity of 
$\left.\mathcal{O}\left(q^{2}+q\right)\right)$. Having settled on an optimum $Q$ value, we focus on the computation of $\mathbf{Y}_{11}$ and $\mathbf{Y}_{21}$ from (8) using the Nyström approximation method (see $[35,36]$ for the extended derivation of $\mathbf{Y})$. We define

$$
\begin{aligned}
& \mathbf{Y}_{11} \triangleq \mathbb{E}\left[\mathbf{H}_{1} \mathbf{H}_{1}^{H}\right]=\widehat{X}_{1} \boldsymbol{\beta}_{s} \widehat{X}_{1}^{H}+\sigma^{2} I_{Q}, \\
& \mathbf{Y}_{21} \triangleq \mathbb{E}\left[\mathbf{H}_{2} \mathbf{H}_{1}^{H}\right]=\widehat{X}_{2} \boldsymbol{\beta}_{s} \widehat{X}_{1}^{H},
\end{aligned}
$$

where $\boldsymbol{\beta}_{s}=\mathbb{E}\left[\bar{S}(\bar{S})^{H}\right]$ with the vectors of the first $Q$ rows of $\widehat{X}$ represented as $\widehat{X}_{1}=\widehat{X}(1: Q$, :) while the remainder $(M N-Q)$ of the row vectors of $\widehat{X}$ also represented as $\widehat{X}_{2}=\widehat{X}(Q+1: M N,:)$. Assuming $\mathbf{Y}_{11}=\mathbf{V}_{11} \boldsymbol{\Theta}_{11} \mathbf{V}_{11}^{H}$ is the eigenvalue decomposition (EVD) of $\mathbf{Y}_{11}$ where the eigenvector is $\mathbf{V}_{11} \in \mathcal{C}^{Q \times Q}$ with $\boldsymbol{\Theta}_{11} \in \mathcal{C}^{Q \times Q}$ as its corresponding diagonal matrix, by letting $\mathbf{V}_{21} \triangleq \mathbf{Y}_{21} \mathbf{V}_{11} \mathbf{V}_{11}^{-1}$, it results in a relaxed, low-rank approximated signal formulation as follows:

$$
\begin{aligned}
& \mathbf{V} \triangleq\left[\begin{array}{c}
\mathbf{V}_{11} \\
\mathbf{V}_{21}
\end{array}\right], \\
& \mathbf{Z}=\mathbf{V} \Theta_{11}^{(1 / 2)} .
\end{aligned}
$$

Alternatively, define $\mathbf{H}_{1} \in \mathbb{C}^{Q \times P}$ to be the first received data submatrices of $Q$ and the remainder of the submatrices as $\mathbf{H}_{2} \in \mathbb{C}^{(M N-Q) \times P}$. Then, (12) can be directly obtained by the following expression:

$$
\mathbf{Z} \triangleq\left[\begin{array}{c}
\mathbf{Y}_{11} \\
\mathbf{Y}_{21}^{H}
\end{array}\right] \mathbf{Y}_{21}^{(-1 / 2)}
$$

In what follows, the sparse representation of $\mathbf{Z}$ that will result in the estimation of the gain-phase errors and the $\mathrm{DOD} / \mathrm{DOA}$ is presented.

\subsection{Sparse-Based Representation for Angle and Gain-Phase} Estimation. Having obtained an approximated signal from (13), the received signal is then represented in the sparse domain so as to exploit its target sparsity in the scenario with gain-phase errors. To begin with, a discretized set, $\left\{\mathbb{S}_{\varnothing}, \mathbb{S}_{\theta}\right\}$, is formed that is made up of all the possible angles of the target as follows $[20,25]$ :

$$
\begin{aligned}
& \mathbb{S}_{\varnothing} \triangleq\left\{\varnothing_{D, v_{1}} \mid v_{1}=0,1, \ldots, V_{1}-1\right\}, \\
& \mathbb{S}_{\theta} \triangleq\left\{\theta_{D, v_{2}} \mid v_{2}=0,1, \ldots, V_{2}-1\right\},
\end{aligned}
$$

where $\varnothing_{D, v_{1}} \leq \varnothing_{D, v_{1}+1}$ and $\theta_{D, v_{2}} \leq \theta_{D, v_{2}+1}$. The targets DOD and DOA can be considered to be $\varnothing_{D, v_{1}}$ entry of $\mathbb{S}_{\varnothing}$ and the $\theta_{D, v_{2}}$ entry of $\mathbb{S}_{\theta}$. Therefore, a formulation of the target steering vector can be obtained as follows:

$$
\boldsymbol{\delta}_{v_{1}, v_{2}}=\mathbf{b}\left(\theta_{D, v_{2}}\right) \otimes \mathbf{a}\left(\varnothing_{D, v_{1}}\right),
$$

which when compiled together forms a dictionary matrix containing all angles within the space angle of $[-\pi, \pi]$ of the target denoted as

$$
\mathscr{D} \triangleq\left[\boldsymbol{\delta}_{0,0}, \boldsymbol{\delta}_{0,1}, \ldots, \boldsymbol{\delta}_{0, Q_{2}-1}, \boldsymbol{\delta}_{1,0}, \ldots, \boldsymbol{\delta}_{V_{1}-1, V_{2}-1}\right] .
$$

Hence, to find the angle of an assumed target, a search is conducted in $\mathscr{D}$ provided the target is within the detection range of the space angle. Granted that in most cases, $M N \gg K$, it can be understood that an obtained solution will be one that presents many zeroes, and only the nonzero solution will lead to an attained estimated angle. Therefore, for any receiver signal under consideration, the resolution lies in obtaining the sparse solution matched against the formulated dictionary matrix. Hence, the signal model in (13) can be reexpressed as follows:

$$
\widehat{Z}=\Gamma \mathscr{D} \bar{S}+\mathbf{E},
$$

which consequently can be formulated into a CS-based problem $[16,19]$ for arriving at the angle estimation problem with gain-phase errors written as follows:

$$
\underset{\mathbf{S}}{\operatorname{argmin}}\|\widehat{Z}-\Gamma \mathscr{D}(\varnothing, \theta) \mathbf{S}\|_{\mathrm{F}}^{2}+\mu\|\mathbf{S}\|_{2,1},
$$

where the sparse matrix $\mathbf{S}$ codified from the scattering coefficient $\bar{S}$ and which also ensure the attainment of a sparse solution is derived as follows:

$$
\begin{aligned}
& \|\mathbf{S}\|_{2,1} \triangleq \sqrt{\sum_{p=1}^{P} \sum_{g=1}^{G} \mathbf{S}_{v, p}^{2},} \\
& \|\mathbf{S}\|_{2,1} \triangleq \sum_{g=1}^{G} \sqrt{\sum_{p=1}^{P} \mathbf{S}_{v, g}^{2}}=\sum_{g=1}^{G}\left\|\mathbf{S}^{g}\right\|_{2},
\end{aligned}
$$

where $\mathbf{G}$ is the discretize grid angles. The $\ell$ norm maintains the accuracy of the sparse reconstruction with $\mu$ being the regulator term that enforces the sparsity of the row. By resorting to the methods proposed by $[37,38]$ to resolve the problem in $(17)$, it results in corrupted estimation since the effect of the gain-phase errors is unknown. Therefore, by initially ignoring the array elements gain-phase errors, we apply a CS-based multiple measurement vector method to estimate the sparse matrix and then compute the array gain-phase error estimation via the gradient descent method. In essence, we propose a fast greedy Nyström CS-based algorithm with gain-phase error estimation algorithm presented in Section 3.3.

3.3. Nyström CS-Based Angle Estimation. In this section, we put together our proposed algorithm. To begin with, the angle estimation for the targets requires the recovery of the sparse vector $\mathbf{S}$ from the obtained signal subspace $\widehat{Z}$, but the exact solution of the optimization problem in (18) is nondeterministic hard. Hence, we employ the CS-based SOMP method $[25,29,39]$ to obtain the sparse matrix and subsequently estimate the DOD/DOA of the targets. SOMP is a greedy approach that adopts a multiple measurement vector technique to reconstruct sparse vectors. This method can increase the number of measurement vectors to attain signal recovery with little information on the sparsity of the signal. Algorithm 1 details the specifics of the proposed algorithm.

At steps 4 and 6 of Algorithm 1, the $Q$ row selection and low-rank approximated signal which applies the FG Nyström theory are estimated. Step 7 applies the SOMP 
(1) Data entry: target number $K$, dictionary matrix $\mathscr{D}$, receiver signal $\mathbf{H}$, and step functionc,

(2) System initialization: iteration $i=0$, assuming $\Gamma=\mathbf{I}$, number of iterations $L$.

(3) While $\mathbf{i} \leq \mathbf{L}-1$ do

(4) Disintegrate $\mathbf{H}$ into submatrices using equation (6)

(5) Compute the value for $Q$ antennas for the submatrices in equation (9).

(6) Compute estimates of $\mathbf{Y}_{11}$ and $\mathbf{Y}_{21}$ using equations (10) and (11).

(7) Obtain approximated signal $\mathbf{Z}$ using equation (12) and transform into sparse domain using equation (17).

(8) Compute estimated sparse matrix using Algorithm 2.

(9) $\mathbf{R}=\left\|\mathbf{V}-\left[\Gamma_{t} \otimes \Gamma_{r}\right] \mathscr{D}(\varnothing, \boldsymbol{\theta}) \widehat{S}\right\|_{\mathbf{F}}^{2}$

(10) Compute $\left(\partial \mathbf{R}\left(\Gamma_{t}, \Gamma_{r}\right)^{H} / \partial \Gamma_{t}\right)$ and $\left(\partial \mathbf{R}\left(\Gamma_{t}, \Gamma_{r}\right)^{H} / \partial \Gamma_{r}\right)$ using equations (23) and (24)

(11) $\Gamma_{t}=\Gamma_{t}-\mathbf{c}\left(\partial \mathbf{R}\left(\Gamma_{t}, \Gamma_{r}\right)^{H} / \partial \Gamma_{t}\right), \Gamma_{r}=\Gamma_{r}-\mathbf{c}\left(\partial \mathbf{R}\left(\Gamma_{t}, \Gamma_{r}\right)^{H} / \partial \Gamma_{r}\right)$

(12) Update the elements of $\Gamma_{t}=0, \Gamma_{t}=0$ with the exception of the maximum

(13) End while

(14) Output: target angles $\varnothing_{k}$ and $\boldsymbol{\theta}_{k}$

(15) End

Algorithm 1: Fast greedy Nyström SOMP with gain-phase error estimation algorithm.

method to estimate and update the sparse matrix $\mathbf{S}$ by choosing the columns that participate in achieving the measurement vector of $\widehat{Z}$ and then proceeds by greedily selecting columns of $\mathscr{D}$ that has an intelligible connection with the successive columns of $\widehat{Z}$ where finally their contribution is updated and iterated. In Algorithm 2, the details of the SOMP algorithm to achieve sparse recovery are presented.

Subsequently, step 7 of Algorithm 1 presents the exploitation of the gradient descent method to achieve and update the estimation of the gain-phase errors of the radar system. Note that the gradient descent method is a simple optimization tool that iteratively finds the local minimum of a function by graduating towards the direction of the steepest descent [40].

Note that at step 6 of Algorithm 2, $\mathscr{D}_{\mathbb{S}_{x}}$ is formulated from the columns of the dictionary matrix with the column indexes obtained from the support set.

With the estimated sparse matrix $\hat{S}$, we compute the gain-phase error vectors $\Gamma_{t}$ and $\Gamma_{r}$ through a derivation of the steepest descent direction by defining the objective function:

$$
\left\{\widehat{\Gamma}_{t}, \widehat{\Gamma}_{r}\right\}=\underset{\widehat{\Gamma}_{t}, \widehat{\Gamma}_{r}}{\operatorname{argmin}} f\left(\Gamma_{t}, \Gamma_{r}\right) \triangleq\|\widehat{Z}-\Gamma \mathscr{D} \widehat{S}\|_{\mathrm{F}}^{2} .
$$

By arriving at the objective function, we can obtain the precise step for each iteration. The derivation of $f\left(\Gamma_{t}, \Gamma_{r}\right)$ can be expressed as follows:

$$
\begin{aligned}
\frac{\partial f\left(\Gamma_{t}, \Gamma_{r}\right)}{\partial \Gamma_{t}}= & -\operatorname{vec}\left\{\widehat{Z}^{H} \mathbf{C} \frac{\partial\left(\Gamma_{t}, \Gamma_{r}\right)}{\partial \Gamma_{t}}\right\}-\operatorname{vec}\left\{\widehat{Z}^{T} \frac{\partial\left[\mathbf{C}\left(\Gamma_{t}, \Gamma_{r}\right)\right]^{*}}{\partial \Gamma_{t}}\right\} \\
& +\operatorname{vec}\left\{\mathbf{C}\left(\Gamma_{t}, \Gamma_{r}\right)^{H} \frac{\partial\left(\Gamma_{t}, \Gamma_{r}\right)}{\partial \Gamma_{t}}\right\} \\
& +\operatorname{vec}\left\{\mathbf{C}\left(\Gamma_{t}, \Gamma_{r}\right)^{T} \frac{\partial\left[\mathbf{C}\left(\Gamma_{t}, \Gamma_{r}\right)\right]^{*}}{\partial \Gamma_{t}}\right\},
\end{aligned}
$$

$$
\begin{aligned}
\frac{\partial f\left(\Gamma_{t}, \Gamma_{r}\right)}{\partial \Gamma_{r}}= & -\operatorname{vec}\left\{\widehat{Z}^{H} \mathbf{C} \frac{\partial\left(\Gamma_{t}, \Gamma_{r}\right)}{\partial \Gamma_{r}}\right\}-\operatorname{vec}\left\{\widehat{Z}^{T} \frac{\partial\left[\mathbf{C}\left(\Gamma_{t}, \Gamma_{r}\right)\right]^{*}}{\partial \Gamma_{r}}\right\} \\
& +\operatorname{vec}\left\{\mathbf{C}\left(\Gamma_{t}, \Gamma_{r}\right)^{H} \frac{\partial\left(\Gamma_{t}, \Gamma_{r}\right)}{\partial \Gamma_{r}}\right\} \\
& +\operatorname{vec}\left\{\mathbf{C}\left(\Gamma_{t}, \Gamma_{r}\right)^{T} \frac{\partial\left[\mathbf{C}\left(\Gamma_{t}, \Gamma_{r}\right)\right]^{*}}{\partial \Gamma_{r}}\right\},
\end{aligned}
$$

where $\mathbf{C}=\operatorname{diag}\{\mathscr{D} \hat{S}\}$. Further, the subgradient of $\left(\partial f\left(\Gamma_{t}, \Gamma_{r}\right) / \partial \Gamma_{r}\right)$ and $\left(\partial f\left(\Gamma_{t}, \Gamma_{r}\right)\right) / \partial \Gamma_{t}$ can be expressed as follows:

$$
\begin{aligned}
\frac{\partial f\left(\Gamma_{t}, \Gamma_{r}\right)}{\partial \Gamma_{t}}= & -2 \mathscr{R}\left\{\operatorname{vec}\left\{\widehat{Z}^{H} \mathbf{C} \frac{\partial\left(\Gamma_{t}, \Gamma_{r}\right)}{\partial \Gamma_{t}}\right\}\right\} \\
& +2 \mathscr{R}\left\{\operatorname{vec}\left\{\left[\mathbf{C}\left(\Gamma_{t}, \Gamma_{r}\right)\right]^{H} \mathbf{C} \frac{\partial\left(\Gamma_{t}, \Gamma_{r}\right)}{\partial \Gamma_{t}}\right\}\right\} \\
= & 2 \mathscr{R}\left\{\operatorname{vec}\left\{\left(\left[\mathbf{C}\left(\Gamma_{t}, \Gamma_{r}\right)\right]^{H} \mathbf{C}-\widehat{Z}^{H} \mathbf{C}\right) \frac{\partial\left(\Gamma_{t}, \Gamma_{r}\right)}{\partial \Gamma_{t}}\right\}\right\}, \\
\frac{\partial f\left(\Gamma_{t}, \Gamma_{r}\right)}{\partial \Gamma_{r}}= & -2 \mathscr{R}\left\{\operatorname{vec}\left\{\widehat{Z}^{H} \mathbf{C} \frac{\partial\left(\Gamma_{t}, \Gamma_{r}\right)}{\partial \Gamma_{r}}\right\}\right\} \\
& +2 \mathscr{R}\left\{\operatorname{vec}\left\{\left[\mathbf{C}\left(\Gamma_{t}, \Gamma_{r}\right)\right]^{H} \mathbf{C} \frac{\partial\left(\Gamma_{t}, \Gamma_{r}\right)}{\partial \Gamma_{r}}\right\}\right\} \\
= & 2 \mathscr{R}\left\{\operatorname{vec}\left\{\left(\left[\mathbf{C}\left(\Gamma_{t}, \Gamma_{r}\right)\right]^{H} \mathbf{C}-\widehat{Z}^{H} \mathbf{C}\right) \frac{\partial\left(\Gamma_{t}, \Gamma_{r}\right)}{\partial \Gamma_{r}}\right\}\right\},
\end{aligned}
$$

where the subgradient of the objective function $f\left(\Gamma_{t}, \Gamma_{r}\right)$ is achieved through the following expressions: 
(1) Data entry: target number $K$, dictionary matrix $\mathscr{D}$,

(2) System initialization: iteration index $L=\mathbf{0}$, Initial residual matrix $\mathbf{V}_{i}=\widehat{Z}$, support set $\mathcal{S}_{x}=\vartheta$

(3) While $\mathbf{L} \leq \mathbf{k}-1$ do

(4) $\left\{\bar{v}_{1}, \bar{v}_{2}\right\}=\operatorname{argmax}_{v_{1}, v_{2}}\left\|\mathbf{V}_{i}^{H} \boldsymbol{\delta}_{v_{1}, v_{2}}\right\|_{1}$

(5) $\boldsymbol{\Lambda}_{x} \longleftarrow \mathcal{S}_{x} \cup\left\{\bar{v}_{1} \mathbf{V}_{i}+\bar{v}_{2}\right\}$

(6) $\widehat{Z}_{i+1}=\mathscr{D}_{\mathcal{S}_{x}} \mathscr{D}_{\mathcal{S}_{x}}^{+} \widehat{Z}$

(7) $\mathbf{V}_{1-1}=\widehat{Z}-\hat{Z}_{i+1}$

(8) $\mathbf{i}=\mathbf{i}+1$

(9) End while

(10) Output: estimated sparse matrix $\widehat{S}$

(11) End

Algorithm 2: SOMP algorithm for angle estimation.

$$
\begin{aligned}
& \frac{\partial f\left(\Gamma_{t}, \Gamma_{r}\right)}{\partial \Gamma_{t}}=\operatorname{diag}\left\{\frac{\partial f\left(\Gamma_{t}, \Gamma_{r}\right)}{\partial \Gamma_{t, 1}}, \frac{\partial f\left(\Gamma_{t}, \Gamma_{r}\right)}{\partial \Gamma_{t, 2}}, \ldots, \frac{\partial f\left(\Gamma_{t}, \Gamma_{r}\right)}{\partial \Gamma_{t, M}}\right\}, \\
& \frac{\partial f\left(\Gamma_{t}, \Gamma_{r}\right)}{\partial \Gamma_{r}}=\operatorname{diag}\left\{\frac{\partial f\left(\Gamma_{t}, \Gamma_{r}\right)}{\partial \Gamma_{r, 1}}, \frac{\partial f\left(\Gamma_{t}, \Gamma_{r}\right)}{\partial \Gamma_{r, 2}}, \ldots, \frac{\partial f\left(\Gamma_{t}, \Gamma_{r}\right)}{\partial \Gamma_{r, N}}\right\},
\end{aligned}
$$

and the gain-phase errors are attained by the following equations:

$$
\begin{aligned}
& \frac{\partial f\left(\Gamma_{t, i}, \Gamma_{r, i}\right)}{\partial \Gamma_{t, i}}=\gamma_{t, i}, \\
& \frac{\partial f\left(\Gamma_{t, i}, \Gamma_{r, i}\right)}{\partial \Gamma_{r, i}}=\gamma_{r, i} .
\end{aligned}
$$

Note that $c(\partial f(\Gamma) / \partial \Gamma)$ is deplored as the step function for every iteration with $c$ as a fixed step.

\section{Analysis of the Algorithm}

4.1. Derivation of Cramér-Rao Lower Bound (CRLB). In this section, the Cramér-Rao bound for the angle estimation with gain-phase error effect for the system under study is derived to analyze the efficiency of the proposed algorithm in addition to observing the bound of the variance of the estimated parameters. The signal model in (5) can be rewritten in a vectorized expression as follows:

$$
\mathbf{h}(p)=\Gamma \mathbf{X} \bar{s}(p)+\mathbf{e}(p)
$$

granted that the target signal is assumed to be uncorrelated with the noise variance $\sigma^{2}$. We further assume that the received signal $\mathbf{h}=\left[\mathbf{h}^{T}(p), \ldots, \mathbf{h}^{T}(P)\right] \in \mathbb{C}^{M N P \times 1}$ has the mean $\boldsymbol{\mu} \in \mathbb{C}^{M N P \times 1}$ with covariance matrix $\Gamma \in \mathbb{C}^{M N P \times M N P}$ which can be written as follows [41]:

$$
\begin{aligned}
\boldsymbol{\mu} & =[\widehat{X} \bar{s}(1), \ldots, \widehat{X} \bar{s}(P)]^{\mathrm{T}}=\Upsilon \mathbf{s}, \\
\boldsymbol{\Lambda} & =\operatorname{blkdiag}\left\{\sigma^{2} \mathbf{I}_{M N_{1}}, \ldots, \sigma^{2} \mathbf{I}_{M N_{P}}\right\},
\end{aligned}
$$

where $\Upsilon=\operatorname{blkdiag}\left\{\widehat{X}_{1}, \ldots, \widehat{X}_{P}\right\} \in \mathbb{C}^{M N P \times P K}$, and $\mathbf{s}=\left[\bar{s}^{T}\right.$ $\left.(p), \ldots, \bar{s}^{T}(P)\right]^{T} \in \mathbb{C}^{P K \times 1}$. Taken that $\Phi=[\varnothing, \theta] \in \mathbb{C}^{1 \times 2 K}$ with $\varnothing \triangleq\left[\varnothing_{1}, \ldots, \varnothing_{k}\right], \theta \triangleq\left[\theta_{1}, \ldots, \theta_{k}\right], \quad \psi=\left[\mathscr{R}\left\{\Gamma_{r} \Gamma_{t}\right\}, \mathrm{I}\left\{\Gamma_{r}\right.\right.$ $\left.\left.\Gamma_{t}\right\}\right] \in \mathbb{C}^{1 \times 4 P}$ and $\boldsymbol{\alpha}=\left[\mathscr{R}\left\{\mathbf{s}^{T}\right\}, \mathrm{I}\left\{\mathbf{s}^{T}\right\}\right] \in \mathbb{C}^{1 \times 2 P K}$, the entire system model can be formulated as $\boldsymbol{\rho}=\left[\boldsymbol{\Phi}, \boldsymbol{\psi}, \boldsymbol{\alpha}, \sigma^{2}\right]^{T}$. The $\mathrm{CRB}$ matrix $\left(e_{\mathrm{CRB}}\right)$ as stated by [42] is obtained as an inverse of the Fisher information matrix (FIM) and is posed as follows:

$$
e_{\mathrm{CRB}}=\mathscr{E}\left[\mathscr{R}\left\{\mathbb{N}^{H} \mathbb{N}\right\}\right]^{-1},
$$

where $\varepsilon=\left(\sigma^{2} / 2\right)$ and $\mathbb{N}=[(\partial \boldsymbol{\mu} / \partial \Phi),(\partial \boldsymbol{\mu} / \partial \psi),(\partial \boldsymbol{\mu} / \partial \boldsymbol{\alpha})$, $\left.\left(\partial \boldsymbol{\mu} / \partial \sigma^{2}\right)\right]$. A direct approach results to $(\partial \boldsymbol{\mu} / \partial \boldsymbol{\alpha})=[\Upsilon, j \Upsilon]$ and $\left(\partial \mu / \partial \sigma^{2}\right)=0$. Take $\left(\partial \hat{x}\left(\varnothing_{\mathrm{k}}, \theta_{\mathrm{k}}\right) / \partial \varnothing_{\mathrm{k}}\right)=\Gamma\left(\left(\partial \mathbf{a}_{m}\left(\varnothing_{\mathrm{k}}\right) /\right.\right.$ $\left.\left.\partial \varnothing_{\mathrm{k}}\right) \otimes \mathbf{b}_{n}\left(\varnothing_{\mathrm{k}}\right)\right), \quad\left(\partial \hat{x}\left(\varnothing_{\mathrm{k}}, \theta_{\mathrm{k}}\right) / \partial \theta_{\mathrm{k}}\right)=\Gamma\left(\left(\partial \mathbf{b}_{n}\left(\theta_{\mathrm{k}}\right) / \partial \theta_{\mathrm{k}}\right) \otimes \mathbf{a}_{n}\right.$ $\left.\left(\varnothing_{\mathrm{k}}\right)\right), k=1, \ldots, K$. Then, deriving $\boldsymbol{\mu}$ on $\Phi$ is expressed as $(\partial \mu / \partial \Phi)=[(\partial \boldsymbol{\mu} / \partial \varnothing),(\partial \boldsymbol{\mu} / \partial \boldsymbol{\theta})]=\left[\boldsymbol{\Lambda}_{1}, \boldsymbol{\Lambda}_{2}\right]$ where

$$
\begin{aligned}
\Lambda_{1}=\left[\begin{array}{ccc}
\frac{\partial \hat{x}\left(\varnothing_{1}, \theta_{1}\right)}{\partial \varnothing_{1}} \bar{s}_{1,1} & \cdots & \frac{\partial \hat{x}\left(\varnothing_{k}, \theta_{k}\right)}{\partial \varnothing_{k}} \bar{s}_{K, 1} \\
\vdots & \ddots & \vdots \\
\frac{\partial \hat{x}\left(\varnothing_{1}, \theta_{1}\right)_{\bar{s}_{1, P}}}{\partial \varnothing_{1}} & \cdots & \frac{\partial \hat{x}\left(\varnothing_{k}, \theta_{k}\right)_{\bar{s}_{K, P}}}{\partial \varnothing_{k}}
\end{array}\right] \in \mathbb{C}^{M N P \times K}, \\
\boldsymbol{\Lambda}_{2}=\left[\begin{array}{ccc}
\frac{\partial \hat{x}\left(\varnothing_{1}, \theta\right)_{1}}{\partial \theta_{1}} \bar{s}_{1,1} & \cdots & \frac{\partial \hat{x}\left(\varnothing_{K}, \theta_{K}\right)}{\partial \theta_{K}} \bar{s}_{K, 1} \\
\vdots & \ddots & \vdots \\
\frac{\partial \hat{x}\left(\varnothing_{1}, \theta_{1}\right)_{\bar{s}_{1, P}}}{\partial \theta_{1}} & \frac{\partial \hat{x}\left(\varnothing_{K}, \theta_{K}\right)_{\bar{s}_{K, P}}}{\partial \theta_{K}}
\end{array}\right] \in \mathbb{C}^{M N P \times K},
\end{aligned}
$$

where $\bar{s}_{k, p}$ is the $k$-th element of $\bar{s}(p)$. 


$$
\frac{\partial \boldsymbol{\mu}}{\partial \psi}=\boldsymbol{\varphi} \triangleq\left[\begin{array}{cccccc}
\frac{\partial \Gamma}{\partial \Gamma_{t 1}} \mathbf{x}_{1}, & \cdots & \frac{\partial \Gamma}{\partial \Gamma_{t \mathrm{M}-\mathrm{m}}} \mathbf{x} \overline{\mathbf{s}}_{1}, & \frac{\partial \Gamma}{\partial \Gamma_{t 1}} \mathbf{x} \overline{\mathbf{s}}_{1} & \cdots & \frac{\partial \Gamma}{\partial \Gamma_{t \mathrm{M}-\mathrm{m}}} \mathbf{x} \overline{\mathbf{s}}_{1} \\
\vdots & \ddots & \vdots & \vdots & \ddots & \vdots \\
\frac{\partial \Gamma}{\partial \Gamma_{t 1}} \mathbf{x}_{\mathrm{p}}, & \cdots, & \frac{\partial \Gamma}{\partial \Gamma_{t \mathrm{M}-\mathrm{m}}} \mathbf{x} \overline{\mathbf{s}}_{P}, & \frac{\partial \Gamma}{\partial \Gamma_{t 1}} \mathbf{x}_{\mathrm{P}} & \cdots & \frac{\partial \Gamma}{\partial \Gamma_{t \mathrm{M}-\mathrm{m}}} \mathbf{x} \overline{\mathbf{s}}_{P},
\end{array}\right] \otimes[1, j]
$$

Then, defining $(\partial \boldsymbol{\mu} / \partial \rho)=[\boldsymbol{\Lambda}, \boldsymbol{\varphi}, \Upsilon, j \Upsilon, 0]$ where $\boldsymbol{\Lambda}=$ $\left[\Lambda_{1}, \Lambda_{2}\right]$, it results in the expression

$$
\begin{aligned}
\mathscr{R}\left\{\mathbb{N}^{H} \mathbb{N}\right\} & =\left[\begin{array}{ll}
\mathbf{J} & 0 \\
0 & 0
\end{array}\right], \\
\mathbf{J} & \triangleq \mathscr{R}\left[\begin{array}{c}
\Lambda^{H} \\
\boldsymbol{\varphi}^{H} \\
\Upsilon^{H} \\
-j \Upsilon^{H}
\end{array}\right]\{[\Lambda, \varphi, \Upsilon, j \Upsilon]\} .
\end{aligned}
$$

From here, we extract $e_{\mathrm{CRB}}$ from $\mathbf{J}$ by applying diagonalization to arrive at the corresponding angle estimation with array gain-phase errors. Granted that $\Upsilon^{H} \Upsilon$ is nonsingular, by defining $\mathbf{L}_{\Lambda}=\left(\Upsilon^{H} \Upsilon\right)^{-1} \Upsilon^{H} \boldsymbol{\Lambda}$ and $\mathbf{L}_{\varphi}=\left(\Upsilon^{H} \Upsilon\right)^{-1} \Upsilon^{H} \boldsymbol{\Lambda}$, the expression of $\mathbf{L}_{\Lambda}^{-1}$ and $\mathbf{L}_{\varphi}^{-1}$ is valid, and hence, we can formulate a transformation matrix as follows:

$$
\mathbf{F}=\left[\begin{array}{cccc}
\mathbf{I} & 0 & 0 & 0 \\
0 & \mathbf{I} & 0 & 0 \\
-\mathscr{R}\left\{\mathbf{L}_{\Lambda}\right\} & -\mathscr{R}\left\{\mathbf{L}_{\varphi}\right\} & \mathbf{I} & 0 \\
-\mathrm{I}\left\{\mathbf{L}_{\Lambda}\right\} & -\mathrm{I}\left\{\mathbf{L}_{\varphi}\right\} & 0
\end{array}\right]
$$

Accordingly, we can express

$$
\left[\begin{array}{c}
\Lambda \\
\varphi \\
\Upsilon \\
\jmath
\end{array}\right] \mathbf{F}=\left[\begin{array}{c}
\left(\Lambda-\Upsilon \mathbf{L}_{\Lambda}\right) \\
\left(\varphi-\Upsilon \mathbf{L}_{\varphi}\right) \\
\Upsilon \\
j \Upsilon
\end{array}\right] .
$$

Then, by orthogonally projecting $\Upsilon^{H}$ onto the null space to obtain $\Pi_{\Upsilon}^{\perp}$, where $\Pi_{\Upsilon}^{\perp}=\mathbf{I}-\Upsilon\left(\Upsilon^{H} \Upsilon\right)^{-1} \Upsilon^{H}$ and $\Upsilon^{H} \Pi_{\Upsilon}^{\perp}=0$, we can deduce that

$$
\begin{aligned}
\mathbf{F}^{H} \mathbf{J F} & =\mathscr{R}\left\{\left[\begin{array}{c}
\boldsymbol{\Lambda}^{H} \Pi_{\Upsilon}^{\perp} \\
\boldsymbol{\varphi}^{H} \Pi_{\Upsilon}^{\perp} \\
\Upsilon^{H} \Pi_{\Upsilon}^{\perp} \\
-j \Upsilon^{H} \Pi_{\Upsilon}^{\perp}
\end{array}\right]\left[\Pi_{\Upsilon}^{\perp} \boldsymbol{\Lambda}, \Pi_{\Upsilon}^{\perp} \boldsymbol{\varphi}, \Upsilon, j \Upsilon\right]\right\}, \\
& =\mathscr{R}\left\{\left[\begin{array}{cccc}
\boldsymbol{\Lambda}^{H} \Pi_{\Upsilon}^{\perp} \boldsymbol{\Lambda} & \Lambda^{H} \Pi_{\Upsilon}^{\perp} \boldsymbol{\varphi} & 0 & 0 \\
\boldsymbol{\varphi}^{H} \Pi_{\Upsilon}^{\perp} \boldsymbol{\Lambda} & \boldsymbol{\varphi}^{H} \Pi_{\Upsilon}^{\perp} \boldsymbol{\varphi} & 0 & 0 \\
0 & 0 & \Upsilon^{H} \Upsilon & j \Upsilon^{H} \Upsilon \\
0 & 0 & -j \Upsilon^{H} \Upsilon & \Upsilon^{H} \Upsilon
\end{array}\right]\right\} .
\end{aligned}
$$

By employing the petitioned diagonal matrix property, we obtain

$$
\begin{aligned}
\mathbf{J}^{-1} & =\mathbf{F}\left(\mathbf{F}^{H} \mathbf{J F}\right)^{-1} \mathbf{F}^{\mathrm{T}} \\
& =\left[\begin{array}{ccc}
\mathbf{I} & 0 & 0 \\
0 & \mathbf{I} & 0 \\
\times & \times & \mathbf{I}
\end{array}\right] \cdot\left[\begin{array}{ccc}
\mathscr{R}\left\{\boldsymbol{\Lambda}^{H} \Pi_{\mathbf{Y}}^{\perp} \boldsymbol{\Lambda}\right\} & \mathscr{R}\left\{\boldsymbol{\Lambda}^{H} \Pi_{\Upsilon}^{\perp} \boldsymbol{\varphi}\right\} & 0 \\
\mathscr{R}\left\{\boldsymbol{\varphi}^{H} \Pi_{\mathbf{Y}}^{\perp} \boldsymbol{\Lambda}\right\} & \mathscr{R}\left\{\boldsymbol{\varphi}^{H} \Pi_{\Upsilon}^{\perp} \boldsymbol{\varphi}\right\} & 0 \\
0 & 0 & \times
\end{array}\right]^{-1} \cdot\left[\begin{array}{ccc}
\mathbf{I} & 0 & \times \\
0 & \mathbf{I} & \times \\
0 & 0 & \mathbf{I}
\end{array}\right] \\
& =\left[\begin{array}{ccc}
\mathscr{R}\left\{\boldsymbol{\Lambda}^{H} \Pi_{\Upsilon}^{\perp} \boldsymbol{\Lambda}\right\} & \mathscr{R}\left\{\boldsymbol{\Lambda}^{H} \Pi_{\Upsilon}^{\perp} \boldsymbol{\varphi}\right\} & \times \\
\mathscr{R}\left\{\boldsymbol{\varphi}^{H} \Pi_{\Upsilon}^{\perp} \boldsymbol{\Lambda}\right\} & \mathscr{R}\left\{\boldsymbol{\varphi}^{H} \Pi_{\Upsilon}^{+} \boldsymbol{\varphi}\right\} & \times \\
\times & \times & \times
\end{array}\right]^{-1},
\end{aligned}
$$

where $\times$ illustrates the irrelevant part in the CRB derivation. Finally, by plugging (31) and (35) into (28) and factoring out all unaffected modes ends in the overall expression

$$
e_{\mathrm{CRB}}=\mathscr{E}\left[\begin{array}{ll}
\mathscr{R}\left\{\boldsymbol{\Lambda}^{H} \Pi_{\Upsilon}^{\perp} \boldsymbol{\Lambda}\right\} & \mathscr{R}\left\{\boldsymbol{\Lambda}^{H} \Pi_{\Upsilon}^{\perp} \boldsymbol{\varphi}\right\} \\
\mathscr{R}\left\{\boldsymbol{\varphi}^{H} \Pi_{\Upsilon}^{\perp} \boldsymbol{\Lambda}\right\} & \mathscr{R}\left\{\boldsymbol{\varphi}^{H} \Pi_{\Upsilon}^{\perp} \boldsymbol{\varphi}\right\}
\end{array}\right]^{-1} .
$$

4.2. Computational Complexity. The proposed algorithm computes $\mathbf{Z}$ circumventing the need for the formation of the entire SCM as it only requires $\mathbf{Y}_{11}$ and $\mathbf{Y}_{21}$ which need $\mathcal{O}(P Q+q)$ and $\mathcal{O}(M N P Q-P Q+q)$ floating-point operations, respectively. Step 4 of Algorithm 2 requires $\mathcal{O}\left(G^{3}+\right.$ $\left.M N G^{2}\right)$ computations for grid discretization and step 9 of Algorithm 1 only needs $\mathscr{O}\left(M N P G+(M N)^{2} G\right)$ floatingpoint operations to estimate the gain-phase error effects. Hence, put all together, the proposed algorithm will necessitate complex-valued flops of $\mathscr{O}\left(G^{3}+M N G^{2}+\right.$ $\left.\left(M N^{2}\right) G+(P Q+q)\right)$. Nonetheless, the methods in $[15,29]$ will necessitate $\mathcal{O}\left(G^{3}+\left(G^{2} M N\right)+\left(G(M N)^{2}\right)+M N P G\right)$ and $\mathcal{O}\left(M^{2} N^{2} P+M^{3} N^{3}+p(M-1)\left(M^{2} N^{3}-M N^{2} K\right)+p\right.$ $\left.(M-1)(N-1)\left(M^{2} N^{2} K-M N K^{2}\right)\right)$ complex-valued flops, respectively, which is expensive than the proposed method.

4.3. Merits of the Proposed Algorithm. In brief, we present a summary of the advantages of our proposed algorithm having compared to the RD-MUSIC [15], sparse-based method [28], and CS-based method [29], in the following:

(1) It efficiently computes and attains paired angle estimation

(2) The proposed estimation algorithm is well suited for reconstructing signals in the presence of a very low number of pulses and signal-to-noise ratio 
(3) It presents a much lower computational load compared to its competitors

(4) In addition to its low computational time, it presents an improved angle and gain-phase error estimation accuracy

(5) The proposed algorithm is much simple, robust to modeling errors, and very effective

The numerical simulation in the next section demonstrates the evidence of these merits.

\section{Simulation Results}

In this section, a chain of computational experiments are conducted to establish the effectiveness and superiority of the proposed algorithm in terms of the root mean square error (RMSE) and compared against RD-MUSIC [15], sparse-based method [28] marked as CS-SOMP, CS-based method [30] marked as SMC, and the Cramér-Rao lower bound (CRLB) benchmark. The array antenna pattern considered is the uniform linear array (ULA) with a separating distance of half-wavelength with equal $M$ transmit and $N$ receive antennas for all numerical examples. We assume that there are $K=3$ completely independent targets with their ground truth angles at $\left(\varnothing_{1}, \theta_{1}\right)=\left(-21^{\circ}\right.$, $\left.21^{\circ}\right),\left(\varnothing_{2}, \theta_{2}\right)=\left(14^{\circ},-9^{\circ}\right)$, and $\left(\varnothing_{3}, \theta_{3}\right)=\left(25^{\circ}, 37^{\circ}\right)$. The matrices of the transmit and receive array gain-phase errors are established stochastically as $\Gamma_{t}=\operatorname{diag}\{1,1,1,0.89$ $\left.e^{j 0.98}, 1.1 e^{j 1.35}, 1.21 e^{j 0.12}, 0.92 e^{j 1.97}, 1.35 e^{j 2.65}\right\}$ and $\Gamma_{r}=\operatorname{diag}$ $\left\{1,1,1.49 e^{j 0.58}, 0.95 e^{j 1.15}, 1.24 e^{j 2.35}, 0.76 e^{j 0.65}, 0.53 e^{j 1.22}, \quad 2.1\right.$ $\left.e^{j 0.89}\right\}$, respectively. Unless otherwise stated details of the simulation parameters are listed in Table 1. Every experiment carried out is conducted on a computer with Matlab R2015a, Intel(R) Core(TM) i5-3230M CPU @ $3.60 \mathrm{GHz}$ 6.00 GB installed memory.

In experiment 1, we investigated the target location ability of the proposed algorithm with $\mathrm{SNR}=5$. From the scatter plot in Figure 2, the cross indicates the ground truth location of the target, whereas the circle represents the estimated angle location. It can be observed that the DODs and DOAs of the target can be estimated and paired accurately.

In the succeeding experiments, we define the RMSE average for an independent run of 400 for the performance evaluation as follows:

$$
\mathrm{RMSE}=\frac{1}{K} \sum_{k=1}^{K} \sqrt{\frac{1}{400} \sum_{\mathrm{mc}=1}^{400}\left|\widehat{\varnothing}_{k}^{(\mathrm{mc})}-\varnothing_{k}\right|^{2}+\left|\hat{\theta}_{k}^{(\mathrm{mc})}-\theta_{k}\right|^{2}},
$$

where mc denotes the number of Monte Carlo simulations and $\left(\widehat{\varnothing}_{k}^{(\mathrm{mc})}, \widehat{\theta}_{k}^{(\mathrm{mc})}\right)$ is the $k t h$ target estimated angle of $\left(\varnothing_{k}, \theta_{k}\right)$, respectively, in the $(\varnothing, \theta)$ direction. Hence, in experiment 2, the estimation performance of the proposed algorithm paired against different values of SNR is conducted. From Figure 3, it is understood that the performance of all competing algorithms suffers in low SNR. However, the proposed algorithm significantly returns improved performance outperforming its competitors even as SNR
TABLE 1: Simulation parameters.

\begin{tabular}{lc}
\hline Parameter & Value \\
\hline Carrier frequency $f_{c}$ & $1 \mathrm{GHz}$ \\
Speed of light $c$ & $3 \times 10^{8} \mathrm{~m} / \mathrm{s}$ \\
Wavelength $\lambda$ & $0.3 \mathrm{~m}$ \\
Pulse number $P$ & 100 \\
Antenna space $d$ & $0.15 \mathrm{~m}$ \\
Number of transmit antenna $M$ & 8 \\
Number of receive antenna $N$ & 8 \\
Grid resolution $\left|\varnothing_{D, v_{1}}-\theta_{D, v_{2}}\right|$ & $0.05^{\circ}$ \\
Angle detection range & $-45^{\circ} \sim 45^{\circ}$ \\
Monte Carlo simulation mc & 400 \\
Number of target $K$ & 3 \\
Signal-to-noise ratio $(\mathrm{SNR})$ & 10 \\
\hline
\end{tabular}

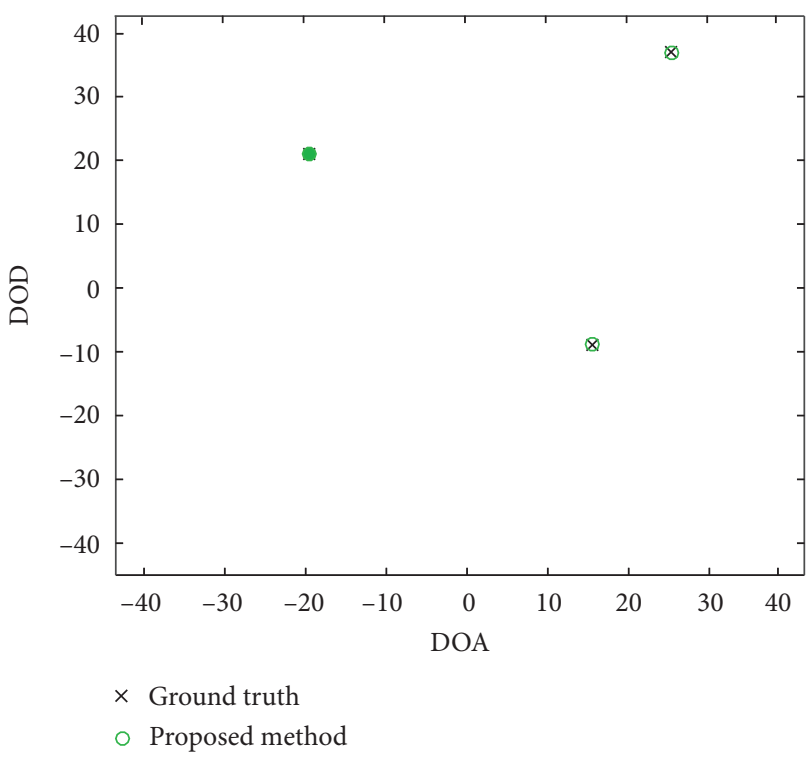

FIGURE 2: Location finding of the proposed algorithm.

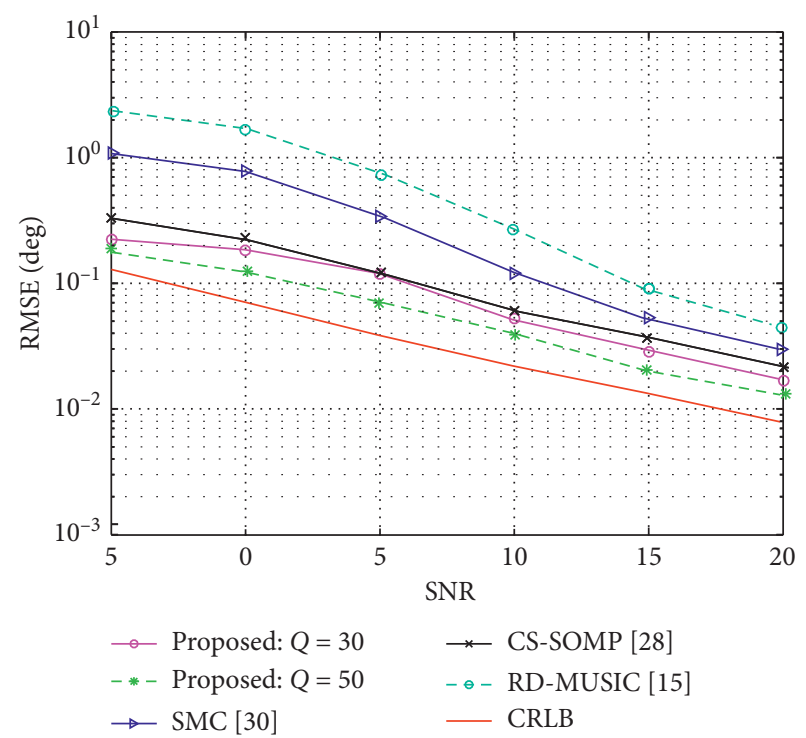

FIgURE 3: RMSE against varying SNR. 


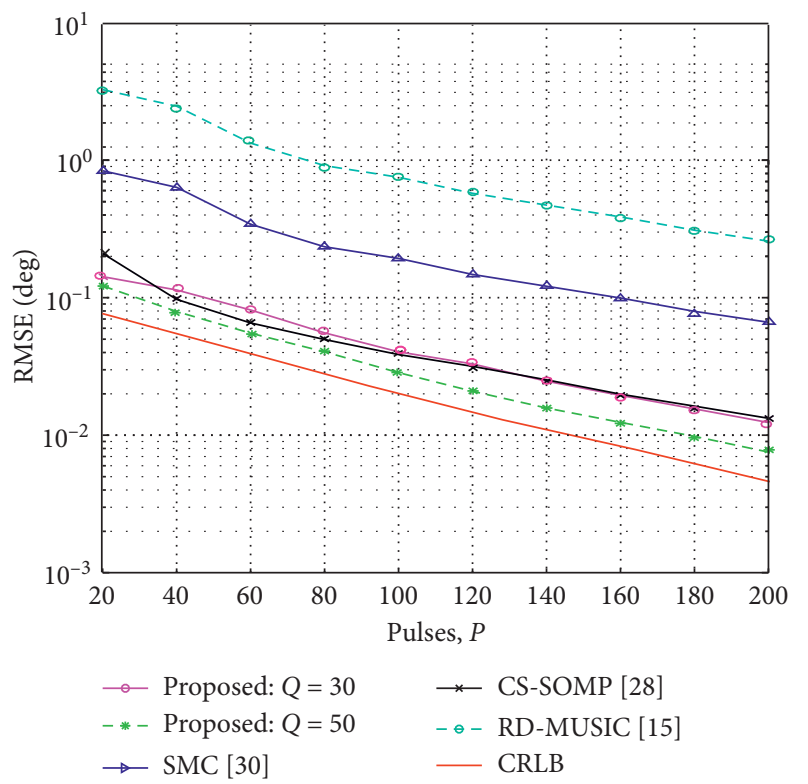

Figure 4: RMSE versus number of $P$.

grows. The proposed method, in addition, demonstrates its ability to approach CRLB. The inference drawn from Figure 3 indicates that the proposed algorithm is less sensitive to the value selection of $Q$. Hence, any optimal $Q$ value selection regardless of the $M=N$ antenna elements and an unstable $P$ is enough to guarantee good results.

Moreover, the exploitation of the sparse field structure of the array measurement further improves the estimated results obtained by the proposed method.

In experiment 3 , we investigated the performance of the proposed algorithm versus different numbers of $P$. From Figure 4 , we can observe that the estimation performance of the proposed algorithm yields improved results as $P$ increases. This is an indication that larger number of $P$ results in better reconstruction performance and in essence, better estimation accuracy. The performance of $Q=30$ falls behind $Q=50$ in all pulse regions and CS-SOMP in the pulse region of 40-80. However, it performed better than CS-SOMP $(P>100)$, SMC, and RDMUSIC. We attribute this inference of the proposed algorithm to its ability to reconstruct signals regardless of the correlation and its inherent robustness to array gain-phase errors.

In experiment 4 , we investigated the angle estimation performance versus different number of antenna elements with large gain-phase errors existence in the array. With the simulation parameters in Table 1 , we assume that 2-to- 4 antenna elements each exhibit large gain-phase errors in the $M$ transmit and $N$ receive antenna array while the errors in the remaining antenna array remain small. From Figure 5, we observed that, as the number of gain-phase errors increases, the performance of the proposed algorithm equivalently improves. This implies that the larger the gain-phase error in the antenna, the better the conversion into nonexistent error and the lower the estimation errors. Figure 4 also indicates that the performance of $Q=30$ is comparable to CS-SOMP but better than RD-MUSIC and SMC.

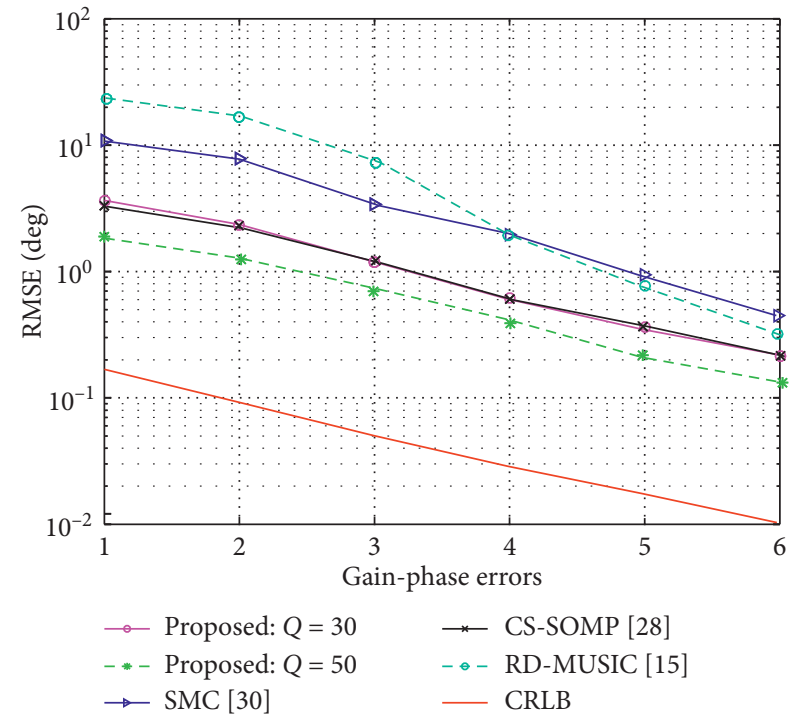

FIGURE 5: Angle estimation performance with varying gain-phase errors.

We investigated the performance of the algorithm against varying numbers of antennas where $M=N$ in experiment 5 .

It is evident from Figure 6 that the performance of the proposed method steadily improves with increasing antenna number. This can be attributed to the diversity gain ability of the MIMO system model implemented.

In experiment 6 , we show the angle estimation performance of the proposed method against varying $K$. We assume that all the $K$ sources are uncorrelated with the noise signal. It is observable from Figure 7 that the proposed algorithm estimation performance steadily improves as SNR increases regardless of the number of $K$. Also since the target 


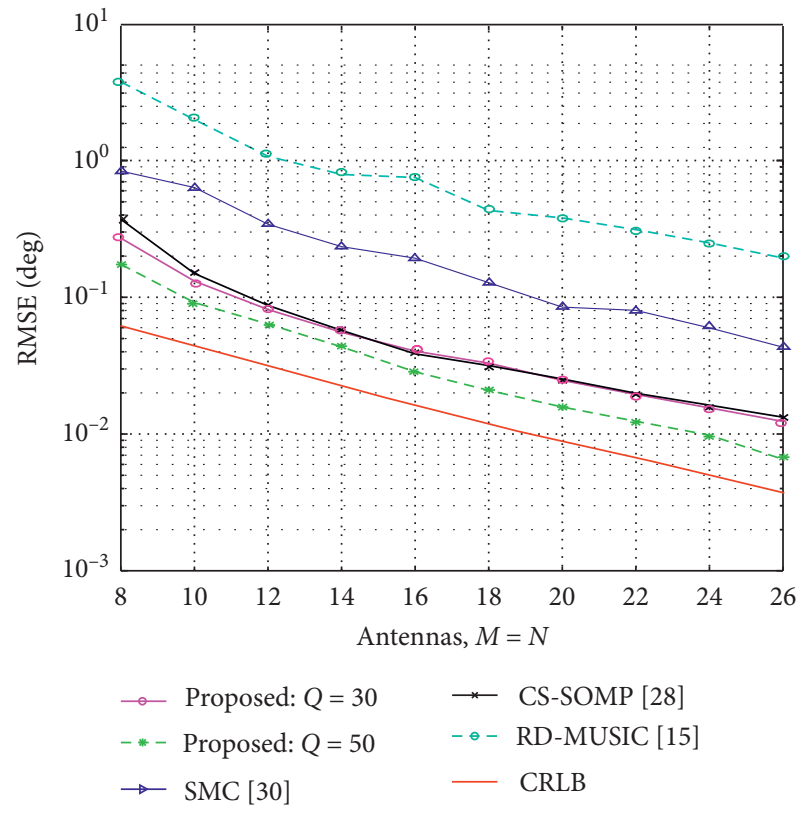

Figure 6: RMSE against number of antennas.

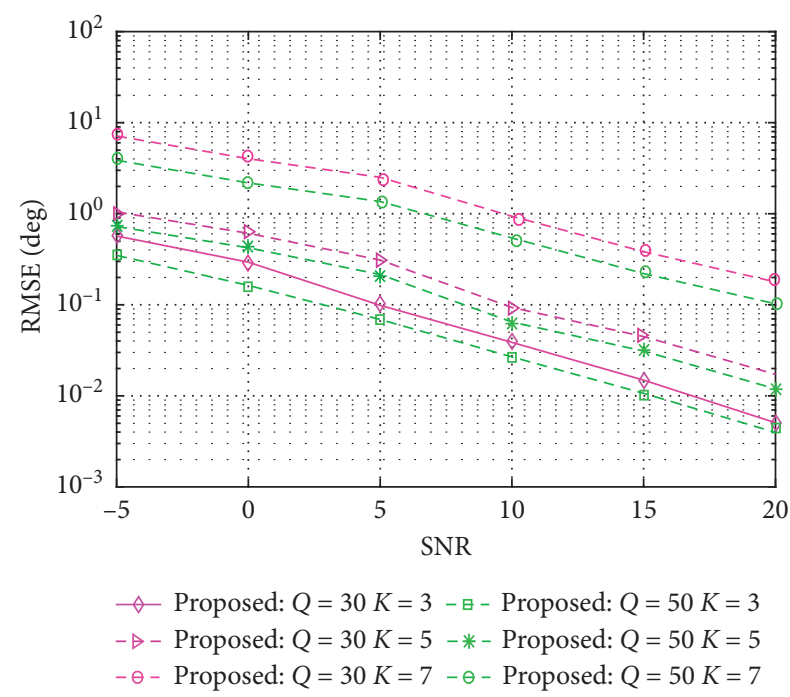

Figure 7: The proposed algorithm estimation performance of varying $K$ against SNR.

sparsity is the main exploitation of the proposed algorithm, experiment 6 affirms the investigation by [43].

Finally, experiment 7 investigates the computational complexity of the proposed algorithm with CS-SOMP, SMC, and RD-MUSIC. We selected $Q=20,30$, and 50 for the proposed algorithm against different $M=N$. The plot in Figure 8 indicates that the proposed algorithm, particularly, $Q=20$, presents a much lower computation time compared to other methods as $M=N$ grows. Another observation identified is the marginal change with increasing $M=N$ of the proposed algorithm computational load, suggesting that the proposed algorithm can be suitable for adaptation into a massive MIMO radar environment.

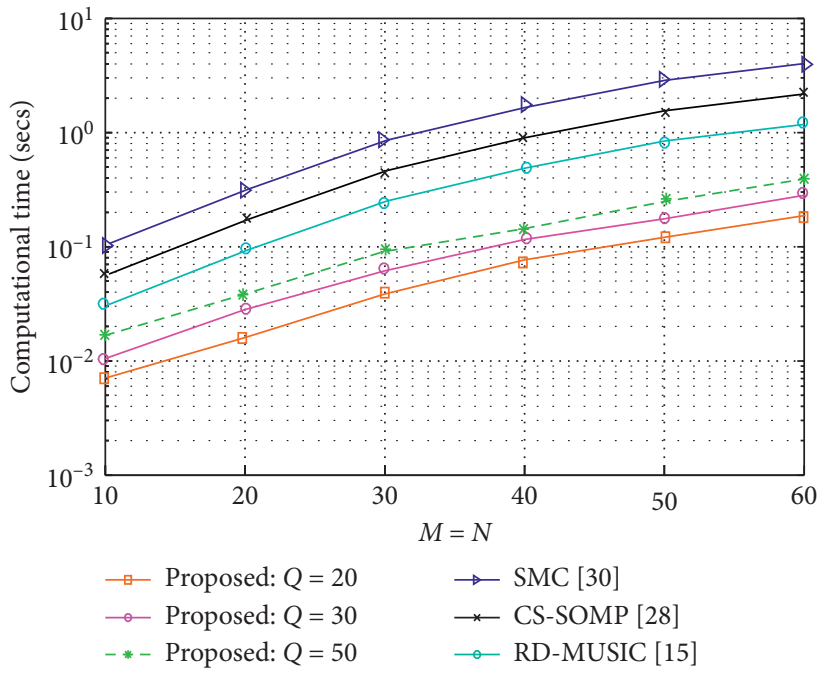

Figure 8: Computational time against number of antennas $(M=N)$.

\section{Conclusion}

A compressed sense method through a fast greedy Nyström approach to achieve a computationally efficient angle estimation with gain-phase error effect for the bistatic MIMO radar is presented in this paper. By introducing a diagonal matrix to formulate the antenna gain-phase errors, the algorithm computes a lower approximation of the received signal which is transformed into a sparse reconstruction problem where its target sparsity is exploited to iteratively estimate the joint DOD/DOA with the unknown gain-phase errors.

The proposed algorithm achieves an improved performance with a much lower computational complexity than its competitors as demonstrated by the numerical experiments. Hence, it can be a desirable application to like-scenarios where computational time, vis-a-vis performance, is a priority. In addition, the theoretical derivation of the CRB which finds the lower bound of the variance of the estimated parameters is obtained. The results from the numerical simulations demonstrate the proposed algorithm improved results against existing algorithms.

\section{Data Availability}

The data used to support the findings of this study are included within the article.

\section{Conflicts of Interest}

The authors declare that there are no conflicts of interest regarding the publication of this paper.

\section{References}

[1] E. Fisher, A. Haimovich, R. Blum, L. J. Cimini, D. Chizhik, and R. A. Valenzuela, "Spatial diversity in radars-models and detection performance," IEEE Transactions on Signal Processing, vol. 54, no. 3, pp. 823-838, 2006.

[2] L. Xu, J. Li, and P. Stoica, "Target detection and parameter estimation for MIMO radar system," IEEE Transactions on 
Aerospace and Electronic Systems, vol. 44, no. 3, pp. 927-939, 2008.

[3] D. R. Fuhrmann and G. S. Antonio, "Transmit beamforming for mimo radar systems using signal cross-correlation," IEEE Transactions on Aerospace and Electronic Systems, vol. 55, no. 8, pp. 171-186, 2008.

[4] G. Cui, H. Li, and M. Rangaswamy, "MIMO radar waveform design with constant modulus and similarity constraints," IEEE Transactions on Signal Processing, vol. 62, no. 2, pp. 343-353, 2014.

[5] K. W. Forsythe and D. W. Bliss, "Waveform correlation and optimization issues for MIMO radar," in Procedings of the Conference Record of the Thirty-Ninth Asilomar Conference onSignals, Systems and Computers, 2005, pp. 1306-1310, Pacific Grove, CA, USA, 2005.

[6] N. Pandey and L. P. Roy, "Convex optimisation based transmit beampattern synthesis for MIMO radar," Electronics Letters, vol. 52, no. 9, pp. 761-763, 2016.

[7] A. Haimovich, R. Blum, and L. Cimini, "MIMO radar with widely separated antennas," IEEE Signal Processing Magazine, vol. 25, no. 1, pp. 116-129, 2008.

[8] A. Zahernia, M. J. Dehghani, and R. Javidan, "MUSIC algorithm for DOA estimation using MIMO arrays," in Proceedings of the 2011 6th International Conference on Telecommunication Systems, Services, and Applications (TSSA), pp. 149-153, Bali, Indonesia, 2011.

[9] H. Yan, J. Li, and G. Liao, "Multitarget identification and localization using bistatic MIMO radar systems," EURASIP Journal on Advances in Signal Processing, vol. 2008, no. 1, Article ID 283483, 2007.

[10] C. Duofang, C. Baixiao, and Q. Guodong, "Angle estimation using ESPRIT in MIMO radar," Electronics Letters, vol. 44, no. 12 , pp. $770-771,2008$.

[11] A. Liu, G. Liao, C. Zeng, Z. Yang, and Q. Xu, “An eigenstructure method for estimating DOA and sensor gain-phase errors," IEEE Transactions on Signal Processing, vol. 59, no. 12, pp. 5944-5956, 2011.

[12] S. Cao, Z. Ye, D. Xu, and X. Xu, "A hadamard product based method for DOA estimation and gain-phase error calibration," IEEE Transactions on Aerospace and Electronic Systems, vol. 49, no. 2, pp. 1224-1233, 2013.

[13] J. Li, X. Zhang, and X. Gao, "A joint scheme for angle and array gain-phase error estimation in bistatic MIMO radar," IEEE Geoscience and Remote Sensing Letters, vol. 10, no. 6, pp. 1478-1482, 2013.

[14] Y. D. Guo, Y. S. Zhang, and N. N. Tong, "ESPRIT-like angle estimation for bistatic MIMO radar with gain and phase uncertainties," Electronics Letters, vol. 47, no. 17, pp. 996-997, 2011.

[15] J. Li, X. Zhang, R. Cao, and M. Zhou, "Reduced-dimension MUSIC for angle and array gain-phase error estimation in bistatic MIMO radar," IEEE Communications Letters, vol. 17, no. 3, pp. 443-446, 2013.

[16] D. L. Donoho, "Compressed sensing," IEEE Transactions on Information Theory, vol. 52, no. 4, pp. 1289-1306, 2006.

[17] A. Gilbert and P. Indyk, "Sparse recovery using sparse matrices," Proceedings of the IEEE, vol. 98, no. 6, pp. 937-947, 2010.

[18] R. G. Baraniuk, V. Cevher, M. F. Duarte, and C. Hegde, "Model-based compressive sensing," IEEE Transactions on Information Theory, vol. 56, pp. 1982-2001, 2010.

[19] E. J. Candes and M. B. Wakin, "An introduction to compressive sampling," IEEE Signal Processing Magazine, vol. 25, no. 2, pp. 21-30, 2008.
[20] P. Chen, C. Qi, and L. Wu, "Antenna placement optimisation for compressed sensing-based distributed MIMO radar," IET Radar, Sonar \& Navigation, vol. 11, no. 2, pp. 285-293, 2017.

[21] D. Malioutov, M. Cetin, and A. S. Willsky, "A sparse signal reconstruction perspective for source localization with sensor arrays," IEEE Transactions on Signal Processing, vol. 53, no. 8, pp. 3010-3022, 2005.

[22] P. Stoica, P. Babu, and J. Li, "SPICE: a sparse covariance-based estimation method for array processing," IEEE Transactions on Signal Processing, vol. 59, no. 2, pp. 629-638, 2011.

[23] J. Chen and X. Huo, "Theoretical results on sparse representations of multiple-measurement vectors," IEEE Transactions on Signal Processing, vol. 54, no. 12, pp. 4634-4643, 2006.

[24] L. Rebollo-Neira and D. Lowe, "Optimized orthogonal matching pursuit Approach," IEEE Signal Processing Letters, vol. 9, no. 4, pp. 137-140, 2002.

[25] E. Baidoo, L. Zhan, and J. Hu, "Kalman filtering method for sparse off-grid angle estimation for bistatic multiple-input multiple-output radar," IET Radar, Sonar \& Navigation, vol. 14, no. 2, pp. 313-319, 2020.

[26] T. Goldstein, C. Studer, and R. G. Baraniuk, "A field guide to forward-backward splitting with a FASTA implementation," 2014, https://arxiv.org/abs/1411.3406.

[27] M. Carlin, P. Rocca, G. Oliveri, F. Viani, and A. Massa, "Directions-of-arrival estimation through Bayesian compressive sensing strategies," IEEE Transactions on Antennas and Propagation, vol. 61, no. 7, pp. 3828-3838, 2013.

[28] L. Liu, X. Zhang, and P. Chen, "Compressed sensing-based DOA estimation with antenna phase errors," Electronics, vol. 8, no. 3, p. 294, 2019.

[29] P. Chen, Z. Cao, Z. Chen, and C. Yu, "Sparse DOD/DOA estimation in a bistatic MIMO radar with mutual coupling effect," Electronics, vol. 7, no. 11, p. 341, 2018.

[30] C. K. I. Williams and M. Seeger, "Using the Nyström method to speed up kernel machines," in Advances in Neural Information Processing Systems, MIT Press, Cambridge, UK, 2000.

[31] A. Nicholas and J. W. Patrick, "Estimating principal components of large covariance matrices using the Nyström method," in Proceedings of the IEEE International Conference on Acoustics, Speech, and Signal Processing (ICASSP), pp. 3784-3787, Prague, Czech, 2011.

[32] P. Drineas and W. M. Micheal, "On the Nyström method for approximating a gram matrix for improved kernel-based learning," Journal of Machine Learning Research, vol. 6, pp. 2153-2175, 2005.

[33] C. Qian, L. Huang, and H. C. So, "Computationally efficient ESPRIT algorithm for direction-of-arrival estimation based on Nyström method," Signal Processing, vol. 94, pp. 74-80, 2014.

[34] A. Farahat, A. Ghodsi, and M. Kamel, "A novel greedy algorithm for Nyström approximation," in Proceedings of the Fourteenth International Conference on Artificial Intelligence and Statistics, vol. 15, pp. 269-277, Fort Lauderdale, FL, USA, 2011.

[35] C. Qian and L. Huang, "A low-complexity Nyström-based algorithm for array subspace estimation," in Proceedings of the 2012 Second International Conference on Instrumentation, Measurement, Computer, Communication and Control, pp. 112-114, Harbin, China, 2012.

[36] C. Fowlkes, S. Belongie, F. Chung, and J. Malik, "Spectral grouping using the nystrom method," IEEE Transactions on Pattern Analysis and Machine Intelligence, vol. 26, no. 2, pp. 214-225, 2004. 
[37] J. W. Choi and B. Shim, "Statistical recovery of simultaneously sparse time-varying signals from multiple measurement vectors," IEEE Transactions on Signal Processing, vol. 63, no. 22, pp. 6136-6148, 2015.

[38] Y. Jin and B. D. Rao, "Support recovery of sparse signals in the presence of multiple measurement vectors," IEEE Transactions on Information Theory, vol. 59, no. 5, pp. 3139-3157, 2013.

[39] J. A. Tropp, A. C. Gilbert, and M. J. Strauss, "Algorithms for simultaneous sparse approximation. Part I: greedy pursuit," Signal Processing, vol. 86, no. 3, pp. 572-588, 2006.

[40] E. M. Dogo, O. J. Afolabi, N. I. Nwulu, B. Twala, and C. O. Aigbavboa, "A comparative analysis of gradient descentbased optimization algorithms on convolutional neural networks," in Proceedings of the 2018 International Conference on Computational Techniques, Electronics and Mechanical Systems (CTEMS), pp. 92-99, Belgaum, India, December 2018.

[41] F. Wen, X. Xiong, and Z. Zhang, "Angle and mutual coupling estimation in bistatic MIMO radar based on PARAFAC decomposition," Digital Signal Processing, vol. 65, pp. 1-10, 2017.

[42] P. Stoica and A. Nehorai, "Performance study of conditional and unconditional direction-of-arrival estimation," IEEE Transactions on Acoustics, Speech, and Signal Processing, vol. 38, no. 10, pp. 1783-1795, 1990.

[43] J. A. Tropp, Topics in sparse approximation, Computational and Applied Mathematics, The University of Texas at Austin, Ph.D. dissertation, 2004. 